\title{
Comparative Analysis of Mesh Generators and MIC(0) Preconditioning of FEM Elasticity Systems
}

\author{
Nikola Kosturski and Svetozar Margenov \\ Institute for Parallel Processing, Bulgarian Academy of Sciences
}

\begin{abstract}
In this study, the topics of grid generation and FEM applications are studied together following their natural synergy. We consider the following three grid generators: Triangle, NETGEN and Gmsh. The quantitative analysis is based on the number of elements/nodes needed to obtain a triangulation of a given domain, satisfying a certain minimal angle condition. After that, the performance of two displacement decomposition (DD) preconditioners that exploit modified incomplete Cholesky factorization $\mathrm{MIC}(0)$ is studied in the case of FEM matrices arising from the discretization of the two-dimensional equations of elasticity on nonstructured grids.
\end{abstract}

Keywords: FEM, PCG, MIC(0), displacement decomposition.

\section{Introduction}

Mesh generation techniques are now widely employed in various scientific and engineering fields that make use of physical models based on partial differential equations. While there are a lot of works devoted to finite element methods (FEM) and their applications, it appears that the issues of meshing technologies in this context are less investigated. Thus, in the best cases, this aspect is briefly mentioned as a technical point that is possibly non-trivial.

In this paper we consider the problem of linear elasticity with isotropic materials. Let $\Omega \subset \mathbb{R}^{2}$ be a bounded domain with boundary $\Gamma=\partial \Omega$ and $\mathbf{u}=\left(u_{1}, u_{2}\right)$ the displacement in $\Omega$. The components of the small strain tensor are

$$
\varepsilon_{i j}=\frac{1}{2}\left(\frac{\partial u_{i}}{\partial x_{j}}+\frac{\partial u_{j}}{\partial x_{i}}\right), \quad 1 \leq i, j \leq 2
$$

and the components of the Cauchy stress tensor are

$$
\tau_{i j}=\sum_{k, l=1}^{2} c_{i j k l} \varepsilon_{k l}(\mathbf{u}), \quad 1 \leq i, j \leq 2
$$

where the coefficients $c_{i j k l}$ describe the behavior of the material. In the case of isotropic material the only non-zero coefficients are

$$
c_{i i i i}=\lambda+2 \mu, \quad c_{i i j j}=\lambda, \quad c_{i j i j}=c_{i j j i}=\mu .
$$

T. Boyanov et al. (Eds.): NMA 2006, LNCS 4310, pp. 7481 2007.

(C) Springer-Verlag Berlin Heidelberg 2007 
Now, we can introduce the Lamé's system of linear elasticity (see, e.g., [2])

$$
(\lambda+\mu) \nabla(\nabla \cdot \mathbf{u})_{i}+\mu \Delta u_{i}+F_{i}=0, \quad 1 \leq i \leq 2
$$

equipped with boundary conditions

$$
\begin{array}{ll}
u_{i}(\mathbf{x})=g_{i}(\mathbf{x}), & \mathbf{x} \in \Gamma_{D} \subset \partial \Omega, \\
\sum_{j=1}^{2} \tau_{i j}(\mathbf{x}) n_{j}(\mathbf{x})=h_{i}(\mathbf{x}), & \mathbf{x} \in \Gamma_{N} \subset \partial \Omega,
\end{array}
$$

where $n_{j}(\mathbf{x})$ denotes the components of the outward unit normal vector $\mathbf{n}$ onto the boundary $\mathrm{x} \in \Gamma_{N}$. The finite element method (FEM) is applied for discretization of (4) where linear finite elements on a triangulation $\mathcal{T}$ are used. The preconditioned conjugate gradient (PCG) [1] method will be used for the solution of the arising linear algebraic system $K \mathbf{u}_{\mathbf{h}}=\mathbf{f}_{\mathbf{h}}$.

\section{MIC(0) DD Preconditioning}

We first recall some known facts about the modified incomplete Cholesky factorization $\operatorname{MIC}(0)$, see, e.g. [46]. Let $A=\left(a_{i j}\right)$ be a symmetric $n \times n$ matrix and let

$$
A=D-L-L^{T},
$$

where $D$ is the diagonal and $-L$ is the strictly lower triangular part of $A$. Then we consider the factorization

$$
C_{\mathrm{MIC}(0)}=(X-L) X^{-1}(X-L)^{T},
$$

where $X=\operatorname{diag}\left(x_{1}, \ldots, x_{n}\right)$ is a diagonal matrix, such that the sums of the rows of $C_{\mathrm{MIC}(0)}$ and $A$ are equal

$$
C_{\mathrm{MIC}(0)} \mathbf{e}=A \mathbf{e}, \quad \mathbf{e}=(1, \ldots, 1) \in \mathbb{R}^{n} .
$$

Theorem 1. Let $A=\left(a_{i j}\right)$ be a symmetric $n \times n$ matrix and let

$$
\begin{aligned}
L & \geq 0 \\
A \mathbf{e} & \geq 0 \\
A \mathbf{e}+L^{T} \mathbf{e} & >0 \quad \text { where } \quad \mathbf{e}=(1, \ldots, 1)^{T} .
\end{aligned}
$$

Then there exists a stable $M I C(0)$ factorization of $A$, defined by the diagonal matrix $X=\operatorname{diag}\left(x_{1}, \ldots, x_{n}\right)$, where

$$
x_{i}=a_{i i}-\sum_{k=1}^{i-1} \frac{a_{i k}}{x_{k}} \sum_{j=k+1}^{n} a_{k j}>0 .
$$

It is known, that due to the positive offdiagonal entries of the coupled stiffness matrix $K$, the $\operatorname{MIC}(0)$ factorization is not directly applicable to precondition 
the FEM elasticity system. Here we consider a composed algorithm based on a separable displacement two-by-two block representation

$$
\left(\begin{array}{ll}
K_{11} & K_{12} \\
K_{21} & K_{22}
\end{array}\right) \mathbf{u}_{\mathbf{h}}=\mathbf{f}_{\mathbf{h}}
$$

In this setting, the stiffness matrix $K$ is spectrally equivalent to the blockdiagonal approximations $C_{\mathrm{SDC}}$ and $C_{\mathrm{ISO}}$

$$
C_{\mathrm{SDC}}=\left(\begin{array}{cc}
K_{11} & \\
& K_{22}
\end{array}\right), \quad C_{\mathrm{ISO}}=\left(\begin{array}{cc}
A & \\
& A
\end{array}\right),
$$

where $A=\frac{1}{2}\left(K_{11}+K_{22}\right)$. This theoretical background of this displacement decomposition (DD) step is provided by the second Korn's inequality [2] Now the $\mathrm{MIC}(0)$ factorization is applied to the blocks of (11). In what follows, the related preconditioners will be referred to as $C_{\mathrm{SDC}-\mathrm{MIC}(0)}$ and $C_{\mathrm{ISO}-\mathrm{MIC}(0)}$, cf., References [245].

\section{Diagonal Compensation: Condition Number Estimate}

The blocks $K_{11}, K_{22}$, and $A$ correspond to a certain FEM elliptic problem on the triangulation $\mathcal{T}$. When there are some positive off-diagonal entries in the matrix, the stability conditions for $\mathrm{MIC}(0)$ factorization are not satisfied. The diagonal compensation is a general procedure to substitute $A$ by a proper $M$-matrix $\bar{A}$, to which the $\mathrm{MIC}(0)$ factorization is then applied. Here, we will restrict our analysis to the case of isotropic DD, i.e., we will consider the piece-wise Laplacian matrix

$$
A=\sum_{e \in \mathcal{T}} A_{e}
$$

where the summation sign stands for the standard FEM assembling procedure. The following important geometric interpretation of the current element stiffness matrix holds (see, e.g., in 3 )

$$
A_{e}=t_{e}\left(\begin{array}{ccc}
\alpha+\beta & -\alpha & -\beta \\
-\alpha & \alpha+1 & -1 \\
-\beta & -1 & \beta+1
\end{array}\right)
$$

where $\theta_{1} \geq \theta_{2} \geq \theta_{3} \geq \tau>0$ are the angles of the triangle $e \in \mathcal{T}, a=\cot \theta_{1}$, $b=\cot \theta_{2}, c=\cot \theta_{3}, \alpha=\frac{a}{c}$ and $\beta=\frac{b}{c}$. Since $|a| \leq b \leq c$, the element-byelement diagonal compensation is mandatory applied if and only if $a<0$. Then, the modified element and global stiffness matrices read respectively as follows

$$
\bar{A}_{e}=t_{e}\left(\begin{array}{ccc}
\beta & 0 & -\beta \\
0 & 1 & -1 \\
-\beta & -1 & \beta+1
\end{array}\right), \quad \bar{A}=\sum_{e \in \mathcal{T}} \bar{A}_{e} .
$$

Note that $\bar{A}_{e} \equiv A_{e}$ if $a \geq 0$. 


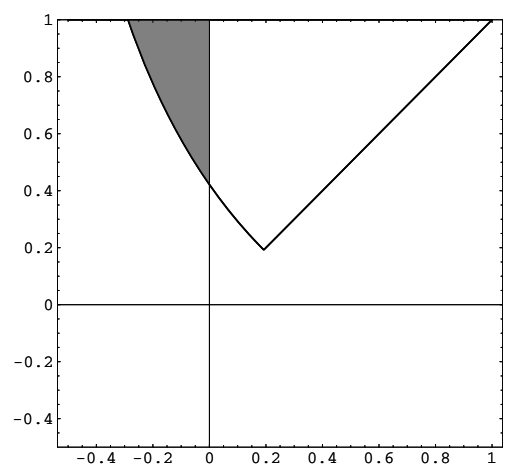

Fig. 1. $\tilde{D}=\left\{\frac{1-t^{2}}{2 t^{2}} \leq \alpha<0, \quad \frac{1-\alpha t^{2}}{t^{2}(1+\alpha)} \leq \beta \leq 1\right\}$

Theorem 2. The relative condition number $\kappa\left(\bar{A}^{-1} A\right)$ is uniformly bounded by a constant, depending only on the minimal angle $\tau$. More precisely

$$
\kappa=\kappa\left(\bar{A}^{-1} A\right) \leq c(\tau)=t^{2}
$$

where $t=\cot \tau$.

Proof. We consider the generalized eigenvalue problem $\bar{A}_{e} \mathbf{u}=\lambda A_{e} \mathbf{u}, \mathbf{u} \neq c \mathbf{e}$ The case $a<0$ corresponds to $(\alpha, \beta) \in \tilde{D}$, see Fig. 1. Straightforward computations lead to $\lambda_{1}=1+\alpha+\frac{\alpha}{\beta}, \lambda_{2}=1$, and therefore

$$
\kappa \leq \kappa_{e}=\frac{\lambda_{2}}{\lambda_{1}}=\frac{\beta}{\alpha+\beta+\alpha \beta} \leq t^{2}
$$

In the final estimate we have used that the maximal value of $\kappa_{e}$ is achieved at the corner point of $\tilde{D},(\alpha, \beta)=\left(\frac{1-t^{2}}{2 t^{2}}, 1\right)$, which completes the proof.

\section{Comparison of Mesh Generators}

In this section, we compare the following three mesh generators:

- Triangle (http://www.cs.cmu.edu/ quake/triangle.html);

- NETGEN (http://www.hpfem.jku.at/netgen/);

- Gmsh (http://geuz.org/gmsh/).

In the previous section we have seen the impact of the minimal angle on the preconditioning. Let us remind also that the minimal angle directly reflects on the accuracy of the FEM approximation as well as on the condition number of the related stiffness matrix. Since a larger minimal angle usually leads to a larger 


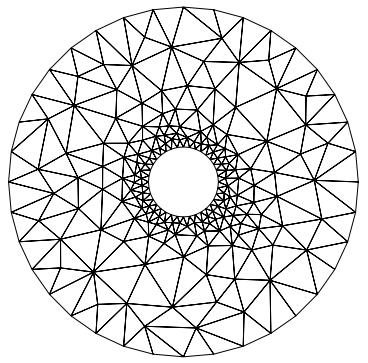

(a)

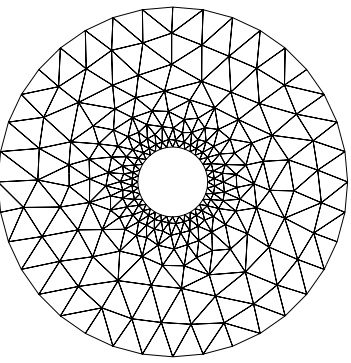

(b)

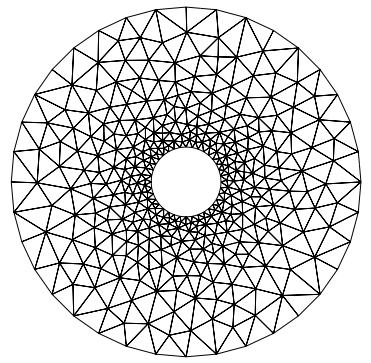

(c)

Fig. 2. Meshes generated by: (a) Triangle; (b) NETGEN; (c) Gmsh

number of elements and nodes in the resulting mesh it is natural to compare the generators based on the numbers of elements and nodes, needed to obtain a mesh with a certain minimal angle.

The domain we chose for this comparison is a disc. The generated meshes, related minimal angles, and numbers of elements and nodes are shown in Fig. 2 and Table 1 respectively.

The results clearly show that Triangle achieved the biggest minimal angle, while also the smallest number of elements and nodes. Note that various parameters may influence the quality of the resulting mesh. Triangle is also the only one of the compared generators that has the minimal angle as a parameter. Triangle's documentation states that the algorithm often succeeds for minimum angles up to $33^{\circ}$ and usually doesn't terminate for larger angles.

\section{Numerical Experiments}

The presented numerical test illustrate the PCG convergence rate of the two studied displacement decomposition algorithms. For a better comparison, the number of iterations for the CG method are also given. Starting with a given coarse mesh, we refine it uniformly connecting the midpoints of each element. Obviously, such a refinement preserves the minimal angle.

Remark 1. The experiments are performed using the perturbed version of the $\mathrm{MIC}(0)$ algorithm, where the incomplete factorization is applied to the matrix

Table 1. Resulting Mesh Properties

\begin{tabular}{llll}
\hline Generator & Minimal angle & Elements & Nodes \\
\hline Triangle & $33.122^{\circ}$ & 386 & 229 \\
NETGEN & $27.4256^{\circ}$ & 440 & 256 \\
Gmsh & $31.8092^{\circ}$ & 688 & 380 \\
\hline
\end{tabular}


$\tilde{A}=A+\tilde{D}$. The diagonal perturbation $\tilde{D}=\tilde{D}(\xi)=\operatorname{diag}\left(\tilde{d}_{1}, \ldots, \tilde{d}_{n}\right)$ is defined as follows:

$$
\tilde{d}_{i}= \begin{cases}\xi a_{i i} & \text { if } a_{i i} \geq 2 w_{i} \\ \xi^{1 / 2} a_{i i} & \text { if } a_{i i}<2 w_{i}\end{cases}
$$

where $0<\xi<1$ is a constant and $w_{i}=-\sum_{j>i} a_{i j}$.

Remark 2. A generalized coordinate-wise ordering is used to ensure the conditions for a stable $\operatorname{MIC}(0)$ factorization.

\subsection{Model Problem in the Unit Square}

We consider first a model pure displacement problem in the unit square $\Omega=$ $[0,1] \times[0,1]$ and $\Gamma_{D}=\partial \Omega$. The material is homogeneous with $\lambda=1$ and $\mu=1.5$, and the right-hand side corresponds to the given solution $u_{1}=x^{2}+$ $\cos (x+y), u_{2}=x^{3}+y^{4}+\sin (y-x)$. A uniform initial (coarsest) triangulation with a mesh size $h=1 / 8$ is used. The stopping criteria used is $\left\|\mathbf{r}^{(k)}\right\|_{\infty} \leq$ $10^{-10}\left\|\mathbf{r}^{(0)}\right\|_{\infty}$.

\subsection{Model Problem in a Disc}

The pure displacement problem with the same given solution and the same stopping criteria as in the the unit square is considered. The computational domain $\Omega$ is a disc with outer radius 1 and inner radius 0.2 . The unstructured initial mesh is shown in Fig. $2(a)$.

\subsection{Computer Simulation of a Pile Foundation System}

We consider the simulation of a foundation system in multi-layer soil media is considered. The system consists of two piles with a linking plate. Fig. 33(a) shows the geometry of $\Omega$ and the related weak soil layers. The mesh is locally refined in areas with expected concentration of stresses, see Fig. 3 (b). The material characteristics of the concrete (piles) are $\lambda_{p}=7666.67 \mathrm{MPa}, \mu_{p}=11500 \mathrm{MPa}$. The related parameters for the soil layers are as follows: $\lambda_{L_{1}}=28.58 \mathrm{MPa}$, $\mu_{L_{1}}=7.14 \mathrm{MPa}, \lambda_{L_{2}}=9.51 \mathrm{MPa}, \mu_{L_{2}}=4.07 \mathrm{MPa}, \lambda_{L_{3}}=2.8 \mathrm{MPa}, \mu_{L_{3}}=$ $2.8 \mathrm{MPa}, \lambda_{L_{4}}=1.28 \mathrm{MPa}, \mu_{L_{4}}=1.92 \mathrm{MPa}$. The forces, acting on the top crosssections of the piles are $F_{1}=(150 \mathrm{kN}, 2000 \mathrm{kN})$ and $F_{2}=(150 \mathrm{kN}, 4000 \mathrm{kN})$.

Table 2. Model Problem in the Unit Square

\begin{tabular}{ccccc}
\hline Mesh & N & CG & ISO-MIC(0) & SDC-MIC (0) \\
\hline 1 & 81 & 67 & 24 & 19 \\
2 & 289 & 129 & 30 & 24 \\
3 & 1089 & 246 & 41 & 32 \\
4 & 4225 & 445 & 59 & 44 \\
5 & 16641 & 853 & 81 & 61 \\
\hline
\end{tabular}




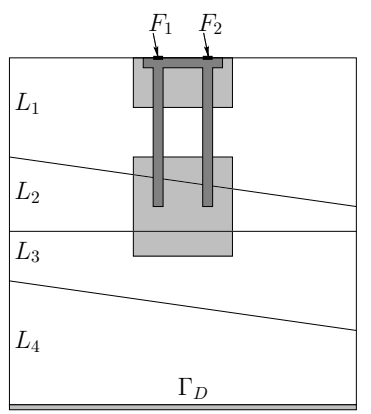

(a)

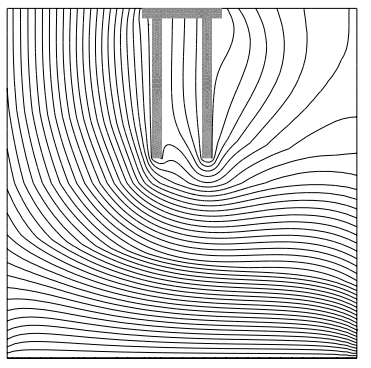

(c)

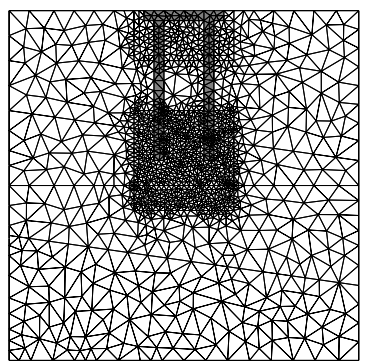

(b)

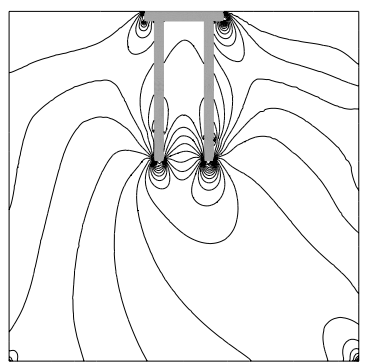

(d)

Fig. 3. Pile Foundation. (a) Geometry of the pile system and the soil layers; (b) The initial mesh with a local refinement; (c) vertical displacements; (d) vertical stresses.

Dirichlet boundary conditions are applied on the bottom side. Table 4 contains the PCG convergence rate for Jacob 1 and the two MIC(0) DD preconditioners. The stopping criteria here is $\left\|\mathbf{r}^{(k)}\right\|_{\infty} \leq 10^{-3}\left\|\mathbf{r}^{(0)}\right\|_{\infty}$. Fig. 3 (c) and (d) show the vertical displacements and vertical stresses respectively.

Table 3. Model Problem in the Disc

\begin{tabular}{ccccc}
\hline Mesh & Nodes & CG & ISO-MIC(0) & SDC-MIC(0) \\
\hline 1 & 229 & 68 & 28 & 27 \\
2 & 844 & 150 & 42 & 40 \\
3 & 3232 & 335 & 62 & 61 \\
4 & 12640 & 712 & 97 & 98 \\
5 & 49984 & 1448 & 159 & 161 \\
\hline
\end{tabular}

\subsection{Concluding Remarks}

The rigorous theory of $\mathrm{MIC}(0)$ preconditioning is applicable to the first test problem only. For a structured grid with a mesh size $h$ and smoothly varying

\footnotetext{
${ }^{1}$ The diagonal of the original matrix is used as a preconditioner.
} 
Table 4. Pile Foundation System

\begin{tabular}{ccccc}
\hline Mesh & Nodes & Jacobi & ISO-MIC(0) & SDC-MIC(0) \\
\hline 1 & 1449 & 725 & 291 & 227 \\
2 & 5710 & 1604 & 412 & 349 \\
3 & 22671 & 3523 & 585 & 527 \\
4 & 90349 & 7468 & 848 & 811 \\
5 & 360729 & 15370 & 1274 & 1334 \\
\hline
\end{tabular}

material coefficents, the estimate $\kappa\left(C_{h}^{-1} A_{h}\right)=O\left(h^{-1}\right)=O\left(N^{1 / 2}\right)$ holds, where $C_{h}$ is the SDC-MIC(0) or ISO-MIC $(0)$ preconditioner. The number of PCG iterations in this case is $n_{i t}=O\left(N^{1 / 4}\right)$. The reported number of iterations fully confirm this estimate. Moreover, we observe the same asymptotics of the PCG iterations for the next two problems, which is not supported by the theory up to now. As we see, the considered algorithms have a stable behaviour for unstructured meshes in a curvilinear domain (see Fig. 2(a)). The robustness in the case of local refinement and strong jumps of the coefficients is well illustrated by the last test problem.

\section{Acknowledgment}

The authors gratefully acknowledge the support provided via EC INCO Grant BIS-21++ 016639/2005. The second author has been also partially supported by the Bulgarian NSF Grant I1402.

\section{References}

1. O. Axelsson: Iterative solution methods, Cambridge University Press, Cambridge, MA, 1994.

2. O. Axelsson, I. Gustafsson: Iterative methods for the Navier equations of elasticity, Comp. Meth. Appl. Mech. Engin., 15 (1978), 241-258.

3. O. Axelsson, S. Margenov: An optimal order multilevel preconditioner with respect to problem and discretization parameters, In Advances in Computations, Theory and Practice, Minev, Wong, Lin (eds.), Vol. 7 (2001), Nova Science: New York, 2-18.

4. R. Blaheta: Displacement Decomposition - incomplete factorization preconditioning techniques for linear elasticity problems, Numer. Lin. Alg. Appl., 1 (1994), 107-126

5. I. Georgiev, S. Margenov: DD-MIC(0) preconditioning of rotated trilinear FEM elasticity systems, Journal of Computer Assisted Mechanics and Engineering Sciences, 11 (2004), 197-209.

6. I. Gustafsson: An incomplete factorization preconditioning method based on modification of element matrices, BIT 36:1 (1996), 86-100. 